\title{
Ethnographic Analogy, the Comparative Method, and Archaeological
}

\section{Special Pleading}

Adrian Currie

Penultimate version, forthcoming in Cambridge Studies in History and Philosophy of Science A

\section{Abstract}

Ethnographic analogy, the use of comparative data from anthropology to inform reconstructions of past human societies, has a troubled history. Archaeologists often express concern about, or outright reject, the practice-and sometimes do so in problematically general terms. This is odd, as (or so I argue) the use of comparative data in archaeology is the same pattern of reasoning as the 'comparative method' in biology, which is a well-developed and robust set of inferences which play a central role in discovering the biological past. In pointing out this continuity, I argue that there is no 'special pleading' on the part of archaeologists in this regard: biologists must overcome analogous epistemic difficulties in their use of comparative data. I then go on to emphasize the local, empirically tractable ways in which particular ethnographic analogies may be licensed.

\section{Introduction}

There are similarities between contemporary and prehistoric human behavior, and so in principle the living can inform us about the dead. This thought underwrites 'ethnographic analogy': the appeal to anthropological reports of contemporary, usually hunter-gatherer behavior, in support of archaeological hypotheses. There are similarities between living organisms and past organisms, and so in principle the extant can inform us about the extinct. 
This thought underwrites the 'comparative method': the appeal to contemporary biological facts to support biological hypotheses about the past.

The comparative method is a well-developed, often quantified and rich set of epistemic techniques which are essential for reconstructing the biological past. By contrast, archaeologists often express concern, suspicion, or outright dismissal of (what they call) ethnographic analogies. Considering that (as we shall see) the two methods represent the same patterns of reasoning, this discrepancy is odd. Is there any reason for archaeologists, and not biologists, to worry about the use of comparative data? Is there 'special pleading' available to archaeologists which might justify such suspicion? I argue that no such case can be made. Just as in biology, the justification or otherwise of the use of comparative data is local and context dependent. My aim is to establish this point, and to make some progress on just what local and context dependent facts might matter.

As we shall see, some archaeologists appear to be wary of ethnographies in principle. For example,

... it can only be constantly restated that analogy does not provide answers, only models, hypotheses and ideas (Hayter 1992, 42).

According to Holly Hayter, ethnographic analogies do not provide evidence, that is, they do not support archaeological hypotheses, but are limited to generating them. In section 4 I will focus on Hayter's discussion of analogy, as it is a rather explicit example of the common attitude I target. In a similar vein, Lewis Binford $(1967,1977)$ also took a conservative view on the role of analogy in archaeology:

Analogy serves to provoke certain types of questions which can, on investigation, lead to the recognition of more comprehensive ranges of order in the archaeological data (Binford, 1967, p10). 
For Binford, then, analogy can 'provoke questions', but does not itself provide 'answers'. When we see the continuities in the use of comparative biological data and comparative ethnographic data, it becomes clear that such positions are untenable. Ethnographic analogies certainly in principle have the goods to provide answers—-that is, evidential support for hypotheses. Recent discussions of ethnographic analogy range from extreme caution or outright rejection (Hiscock 2007, Rednarek 2012, McCall 2007, Barrocall 2011), to more nuanced discussions (Gonzalez-Urquijo et al 2015, Ravn 2011, Whittaker \& Tushingham 2014). My aim is to establish a view on the nuanced end: there is no outright rejection or acceptance of ethnographic analogy to be made, rather, for each case the devil is in the details.

I'll first introduce the comparative method with a paleoanthropological case study (section 2), followed by a similar introduction to ethnographic analogy (section 3). In section 4 I argue against archaeological 'special pleading', that is, there is nothing different, as a matter of epistemic principle, between the biologist, paleoanthropologist, nor archaeologist when drawing such analogies. The interesting question, then, is under what conditions such inferences are licensed.

Appropriately, then, I will discuss what is required to vindicate or damn a particular use of ethnographic analogy. In sections 4 and 5 I note that both ontic and epistemic issues can plague particular applications of comparative data. We must examine the strength and stability of our access to information, and investigate the properties of the systems we are examining: do they behave in a sufficiently regularly to support the inductions comparative data requires? I argue that even in troubling cases, where our information is poor and the systems behave irregularly, ethnographic evidence can still play an important role as one line of evidence involved in reconstructing the cultural past. I use recent work by Christine VanPool (2009) to illustrate how piece-meal, multi-leveled analyses of archaeological remains, drawing on ethnographic information, can lead to rich, well supported hypotheses. 
Note that I am restricting myself to epistemic similarities and differences between archaeology and other sciences. There are important social, technological and financial differences which matter for how these sciences are practiced, but here I will focus on the patterns of reasoning involved.

\section{Hobbits \& Hippos}

In this section I illustrate the comparative method with a paleoanthropological case study. $\mathrm{H}$. floresiensis were a surprising addition to the hominid family tree. Around 13 individuals were found at a single site on the Indonesian island of Flores, which they inhabited up until around 14,000 years ago (Brown et al 2004, Moorwood et al 2005). Their most striking feature is diminutive size—adults reach a paltry 1 meter tall—earning them the inevitable 'hobbit' epithet. In addition to their stature, they also sport 'primitive" features: low encephalization (that is, brain-size/body-size ratio), arboreal adaptations and incomplete bipedalism. H. floresiensis' taxonomic grouping is mysterious: do their features signal a remarkable story of late hominid evolutionary adaptability, or a remarkable story of early hominid radiation and survival? Are the hobbits late hominids gone dwarf, or the last remnant of a hitherto unknown migration of early hominids? These hypotheses provide contrasting explanations of $\mathrm{H}$. floresiensis' traits, which illustrate an essential distinction in the comparative method.

By the 'early hominid' hypothesis, hobbits are the ancestors of a primitive hominid radiation out of Africa, perhaps Homo habilis (Jungers 2009, Wong 2009). H. floresiensis and $H$. habilis share traits: they are small, low in encephalization, walk stooped, and suit partially arboreal lifestyles. By this hypothesis, hobbit traits are the result of retained, ancestral features. They are

\footnotetext{
${ }^{1}$ Here, 'primitive' is certainly no insult to $\mathrm{H}$. floresiensis, rather that some of its traits are associated with the base of the hominid line.
} 
homologues. Two traits are homologous when they are inherited from a common ancestor ${ }^{2}$. By this hypothesis, then, $H$. floresiensis and $H$. habilis' traits signal their ancestral relatedness and their similarity is explained in terms of that ancestry. What's wrong with the 'early hominid' theory? There is no evidence of habiline hominids radiating into Asia: it was the taller, upright and more highly encephalized $H$. ergaster, $H$. erectus and $H$. heidelbergensis who took the hominid torch out of Africa.

The 'late hominid' theory places the divergence between $\mathrm{H}$. floresiensis and the hominid line much later-by this theory their ancestors were erectine and their problematic traits are adaptations to their island environment (Argue et al 2009). Like the pygmy elephants of Flores, the hobbits could be insular dwarves. By this hypothesis, the relationship between the traits of $H$. habilis and $\mathrm{H}$. floresiensis is homoplastic, rather than homologous: the hobbits did not inherit their low encephalization, but rather it evolved via island dwarfism. Homoplastic traits are convergent: rather than tracing ancestry, they trace evolutionary pressure or other influences ${ }^{3}$. What's wrong with the 'late hominid' theory? Standard models of dwarfism do not predict some hobbit features (Martin et al $2006^{4}$, Jungers et al 2009). For instance, it is thought that insular dwarfism is expressed developmentally via shorter growth periods. The difference between a pygmy elephant and a whopper, by this line, is growing time. However, different parts of the body complete growth earlier than others-specifically, brain development completes earlier than body growth. If dwarfism is the result of less growing time, then we should expect dwarves to be more encephalized than their bulky cousins, as the brain had time to mature while the body's growth was cut short. On this model, $H$. floresiensis' brain should be twice the actual size.

\footnotetext{
${ }^{2}$ This is a version of a taxic definition of homology, definitions of homology are highly contentious (see, for instance, Brigandt \& Griffiths 2007, Currie 2014, Hall 2003, Ramsey \& Paterson 2012), but this does not affect the nature of the inference considered here.

${ }^{3}$ Like homology, 'homoplasy' definitions are contentious (see Currie 2014, Pearce 2012, Powell 2012)— but again, this need not concern us now.

${ }^{4}$ Note that Martin et al do not endorse an early hominid model, but rather argue that the features are pathological: 'H. florersiensis' are H. sapiens. See also Jacob et al 2006.
} 
Which hypothesis is more likely: are the hobbit traits inherited homologues, or homoplastic? Were they habiline or erectine? Most obviously, this depends on whether a habiline 'ghost' radiation or an erectine dwarf with hobbit-like features is more plausible. However, there is more to this than meets the eye-let's start with the 'early hominid' hypothesis.

The 'early hominid' hypothesis involves what has been called a phylogenetic (or homologous) inference (see Levy \& Currie 2014, Currie 2015). In such inferences, common features are taken to be indicative of common ancestry, or common ancestry is taken to be evidence of common traits. In this case we infer from the similarities between early hominids and $H$. floresiensis to their having a shared ancestry. An example of the latter would be to appeal to other facts about early hominids to infer further hobbit traits, say that $\mathrm{H}$. floresiensis used the stone-flake based Olduwan tool-set on the basis of their (presumed) habiline ancestors using them. The success of such inferences turn on:

(1) How similar are the traits, and are they similar in relevant respects 5 ? Obviously, brute quantitative similarity has its part to play, but typically, biologists prefer some traits more than others. For instance, traits that are less likely to be affected by selection pressure. Traits related to climbing, for instance, are frequently excluded from reconstructions of monkey phylogenies, as these are highly likely to be homoplastic, and thus too noisy for taxonomic purposes (see Hall 2007).

(2) How labile are the traits? That is, over evolutionary time, should we expect the trait in question to remain stable, or change? Traits under intense and steady maintenance (or 'stabilizing') selection are likely to remain stable. Moreover, canalized 'generatively entrenched' (Wimsatt 1986) traits will also resist change. Others are more labile, and thus problematic. The dramatic changes in hominid brain size over our evolution suggests that

\footnotetext{
${ }^{5}$ Remane (1952) provides five criteria for identifying homologues. Some of these stretch the notion of 'similarity' somewhat: i.e., some depend on the relative positions of the homologues rather than similarity in character states. I take being similar in 'relevant respects' to capture these non-character-state-based notions.
} 
encephalization is labile in hominids, and this underwrites caution about inferring ancestry from that trait alone.

(3) What is the temporal distance? The amount of time between homologues matters for the stability of homologous inferences. For short distances, labile traits could remain stable, whereas over long distances only the most entrenched will.

And so, in addition to the plausibility of a habiline ghost lineage, the evidential support for the 'early hominid' hypothesis depends on the relevant similarity and lability of the traits in question. Let's move to the 'late hominid' hypothesis.

Recall that, by the 'late hominid' hypothesis, $H$. floresiensis were of erectine stock, but evolved shorter stature (and other traits) while adapting to the environment of Flores. As opposed to inferring across ancestry, we here appeal to a model which links particular traits to certain adaptive environments in a particular developmental context. That is, the inference relies on a model claiming that, for hominids, adaptation to island environments could plausibly lead to the hobbit's traits (Currie 2013). The objection to the late hominid hypothesis just is that the model of island dwarfism does not do this. So, how is that model sanctioned?

Weston \& Lister (2009) appeal to other mammalian dwarves to test this developmental model, suggesting that $\mathrm{H}$. floresiensis could have been erectine after all (see Lieberman 2009 for a summary). The key is noticing that the model of island dwarfism does not merely predict high encephalization in hobbits, but across a range of island-dwelling lineages. Assuming that those lineages are relevantly hobbit-like, they can be used to test the model. Weston \& Lister do just this, comparing two lineages of extinct pygmy hippopotamus and an extinct pygmy elephant to full sized variants. Happily for the 'late hominid' hypothesis, body size and encephalization in those lineages bucks the model's trend. This shows that dwarfism is not always expressed via the retardation of later ontogenetic processes-there must be some processes by which brain growth decreases more than body growth. Montgomery \& Mundy (2013) have suggested that 
dwarfism could occur via the gestation period remaining fixed, but fetal development slowing. This could cause early developing traits such as brains and teeth to be more affected than late developing traits. Their model was also supported by an analogue: appeal to the diminutive teeth-size to body-size ratio of pygmy marmosets ${ }^{6}$.

And so, some inferences rely on models which apply to more than just our target-and appealing to these other cases can serve to test and refine the model. In a comparative context, these typically take the following schematic form:

Across phylogenetic group $x$ (in environment $w$ ), feature $y$ correlates with feature $z^{7}$.

For instance, Martin et al's model claimed that, across mammals, dwarfism correlates with increased encephalization. We can test such models by examining members of $x$ in $w$ with feature $y$-pygmy hippos dwelling in island environments in this case; the model is undermined if $z$ is not present. How effective is this? This depends on:

(1) What is the phylogenetic distance between the traits? Lineages with similar developmental systems are more likely to respond similarly to selective or other environmental pressures than those with different developmental systems. Phylogenetic relatedness is a proxy for this. Martin et al, for instance, could complain that their model is only supposed to be applicable to hominid dwarfs, not Afrotheria. For this to bite, they would need to make it plausible that the two phylogenetic groups are likely to diverge in that respect ${ }^{8}$.

\footnotetext{
${ }^{6} \mathrm{~A}$ broader approach is taken by Bromham \& Cardillo (2007). They test the 'island rule', that larger animals lose size and smaller animals gain size on islands, across many primates. They find that the hobbit's gross body size fits within the island rule's range.

${ }^{7}$ See Currie's (2015) parallel discussion of 'bracketed models' for more.

${ }^{8}$ Currie (2013) calls this 'scope'.
} 
(2) How similar are the relevant features ${ }^{9}$ ? In this case, we are concerned with testing the ontogenetic expression of island dwarfism. For instance, Montgomery's appeal to pygmy marmosets could be undermined as they evolved in the Amazon Basin, not on an island. Although both they and Weston \& Lestor's hippos are dwarfs, it could be that pygmy marmosets are not dwarfs by the relevant cause $e^{10}$.

(3) Number of data points: how many independent couplings of $y$ and $z$ are there in $x$ ? Taken alone, Weston \& Lestor's evidence shows that Martin et al's model does not always apply, and so dwarfism is possible in $\mathrm{H}$. floresiensis—but we would need to see more examples, and across a wider range of the relevant phylogenetic group (particularly primates), to say anything stronger.

And so, we can identify two general kinds of comparative inference. The first, homologous inference, either infers traits from ancestry, or ancestry from traits. The second, homoplastic inference, supports models that couple features (sometimes traits to other traits, sometimes traits to environments) by appealing to analogues as data points. One critical difference is that homologous inferences are token-level or individual, while homoplastic inferences are type-level. That is to say, homologous inferences are concerned only with the individual lineage containing the homologues: the inference follows a line of ancestry. In contrast, homoplastic inferences consider the case as an instance of a particular class—-the analogues are unified via a model coupling the lineages' features. Homoplastic traits are instances of a type of event; homologous traits are parts of an historical individual.

I hope it is obvious that the applicability of these two inference patterns are sensitive to context. Although we can discuss what the licence depends on, there is nothing to say about whether homologous or homoplastic inferences are licenced overall. The licence of the

\footnotetext{
${ }^{9}$ Currie (2013) call this 'grain'.

${ }^{10}$ I doubt this: the Amazon Basin quite possibly is an island for ecological purposes, and moreover it is hard to see how a difference in the cause of the selection pressure could affect how the trait is expressed ontogenetically.
} 
inferences depends on the factors I identified, and those factors are local: the temporal distance, the similarity of the traits, the lability, and so forth. It is time to turn to archaeology. As we shall see, ethnographic analogy follows just the same structure as we have just seen for the comparative method.

\section{Stones \& Shaman}

Painted images on natural stone, 'rock' or 'parietial' art, are widespread human artifacts that provide enticing, but ambiguous, insights into the lifeways of those who made them. Archaeological studies of rock art have focused on European and South African cases, but have drawn on ethnographic studies as wide spread as Australia, North America and Papua New Guinea in addition to African sources (see Chippendale \& Taccon 1998). There are, essentially, two questions about rock art. First, what explains rock art? How does it fit into a chronology of human cultural development? Second, and relatedly, what does rock art signal? What can it tell us about past human cultures? Answers to the first question inform the second, and support reconstructions. For instance, the cultural-historical archaeology of the 1950 s and earlier understood the phenomenon in terms of innate human creativity: rock art was an expression of feelings. As it was assumed that such expressions were specific to cultures, they were taken to track chronology and ethnicity (Beroccal 2011). For the 'new archaeologists', by contrast, making rock art was an adaptive behavior, in the business of transmitting important information (Conkey \& Hastorf 1990). If that is right, then rock art can signal past social structures. The functionalists, structuralists and Marxists of the 6os and 70s emphasized rock art's role in maintaining social order. Here, shamanism came to the fore (Lewis-William 1995).

Two contexts matter here: South Africa and Europe. Since the 1970s South African archaeologists had more interest in, and respect for, indigenous people. Where previously a kind of euro-centrism saw hypotheses about Paleolithic European rock art transported into African 
contexts, this stream was reversed. Some indigenous African people, the San in particular, have shamanistic practices (but do not create rock art). A key part of shamanism is the use of motifs to symbolize spiritual information. Such motifs are expressed in story and dance-and, when rock art and shamanism overlap—rock art. For South African archaeologists, then, these motifs became a bridge between contemporary shamanistic practices and rock art-and thus from the art to the ancient artists themselves. And so, although the modern San do not themselves create rock art, it is thought that there is both cultural and environmental continuity between them and the ancient, rock-art producing cultures archaeologists want to understand, and that this is sufficient to justify using San shamanistic practice to inform interpretation.

Researchers now interpret specific panels in terms of well-documented symbols of potency, metaphors of trance experience, significant human and animal postures, entopic phenomena, and hallucinations experienced by San shamans. As this work proceeds, we learn more and more about the 'syntax' and 'vocabulary' of the art and are thus able to 'read' increasingly complex painted texts. Each elucidation deepens our understanding of San thought and religious experience and so provides hitherto unattainable insights into the ideology of the now-extinct painters (Lewis Williams 1989 pp166).

Detailed studies of San ethnographies, then, provide the language of shamanistic motif, which is then applied to the rock art in question. This is an example of what archaeologists call a 'direct' analogy:

Ethnographic information can also be direct, when both the archaeological and ethnographic contexts share a common geographic setting and a potential cultural connection exists between them (Berrocal 2011, pp 6).

An important part of the justification here is the hypothesized continuity between the contemporary San, or near-contemporary in the case of $19^{\text {th }}$ Century ethnographic reports, and the rock artists 3,000 years earlier. Further justification is provided by similarities between the rock art and shamanistic stories, roughly, the capacity of shamanistic motifs to explain otherwise 
baffling features of rock art (Lewis-Williams' 1989 discussion of the meaning of Elan imagery is a striking example).

As I make explicit in section 4, there are structural similarities between the direct analogy connecting the San and ancient African artists, and the homologous inference between $\mathrm{H}$. floresiensis and its habiline ancestors. Both infer, on the basis of relevant similarities, along lines of ancestry. The inferred continuity between cultural groups on the one hand, and between phylogenies on the other, both underlie the reconstruction.

And so, in South Africa, shamanism is used to explain the features of ancient rock art and is supported by the claim that San are a cultural/geographically continuous people with the rock artists. These ideas were then transplanted into Europe, where the inference took on a rather different character (note that the claims that the San can inform interpretation of South African rock art, and that the same ethnographies can inform European rock art are different, and not mutually exclusive).

In Europe, the shaman hypothesis received a neuro-psychological spin (Lewis-Williams 1991, 2004, Lewis-Williams \& Dowson 1988). A long-standing puzzle in European rock art is the meaning behind 'entropic' (that is, simple patterned rather than overtly depictive) motifs. It is suggested that these are caused by shamans directly representing hallucinogenic experiences on the rock. There are regularities in human perception during altered states of consciousness, and these in combination with Shamanistic practices are taken to explain Paleolithic rock art.

This is an example of an 'indirect' analogy: although there is no continuous cultural or geographical connection between the San and European rock artists, it is nonetheless thought that the connection between rock art and Shamanism in a South African context can be transported into Europe in light of human psychological continuities. As evidence once more we have the level of similarity between the rock art (these are sometimes referred to as 'formal 
analogies') and the capacity of the rock art to explain otherwise befuddling aspects of European rock art, such as entropic motifs.

And so, two different inferences are performed. First, a direct analogy is drawn between San ethnographies and South African rock art; the 'language' of contemporary San motif expressed in story, dance and ritual is extended to the rock art. Second, an indirect analogy is drawn between shamanistic practices and European rock art. Here, a model connecting shamanism to rock art is used, partly licensed by an underlying psychological model, by similarities between rock art in Europe and South Africa, and the success of the model in explaining otherwise strange features of the motifs.

The suggestion that Paleolithic art is explicable via analogy with the San is similar to the hypothesized relationship between $\mathrm{H}$. floresiensis and other island dwarfs. In both cases, the target is taken to be a token of a type: the former is an instance of shamanistic motif, the latter an instance of island dwarfism. Seen in this light, the regularities applying to these instances (the meaning of entropic motifs, or the developmental models of dwarfism) are applicable to the targets.

The use of ethnographies to inform rock art interpretation has been heavily criticized, both in its direct and indirect application. These objections are examples of the more general charges I discuss in section 4. Rednarik (2012) puts this all rather starkly:

... I am most pessimistic about our prospects in most of these areas, and in particular, I perceive very little scientific benefit in most traditional [ethnographic] approaches to rock arts. It is not the role of true science to create, reinforce and perpetuate mythologies about the way the world is. Rednarik (2012) p 224

First, data about the analogue itself has come under fire. Characterizations of 'shamanistic' practices in South Africa are largely drawn from San ethnographies and $19^{\text {th }}$ Century ethnohistories of the /Xam (McCall 2007). The trustworthiness of this information has been 
questioned, both considering its antiquity and possible biases (Bahn 1997, Solomon 1998). As Berrocal puts it:

... [such ethnographies] might privilege the vision of a specific individual or group of individuals over the rest of the group, by focusing on the one particular meaning available. Meaning is polysemous and the relation between meaning and material sign is not univocal within the same social group. Knowledge may be unevenly distributed inside the social group. Therefore, there is the danger that ethnographic records might be biased, masking differences in knowledge and/or power (12).

Not only might ethnographies be marred due to non-ideal data gathering, but Berrocal emphasizes that multiple meanings frequently exist amongst a group that are obscure to outside observers. The difficulty of capturing this diversity leads to biases. A second line of attack tackles the idea that 'shamanism' represents a unified cultural 'type' that one may infer across (see Layton 2000). McCall argues forcefully for this:

In seeking to interpret rock art using universal features of cosmology and religious practice, it is highly generalizing. It denies the importance of regional historical and social contexts in determining symbolic practices. In seeking singular meanings for inherently polysemous symbols, it clearly lacks the kind of multivocality that has become a key feature of post-processual approaches (226).

Roughly, according to McCall, if hunter-gatherer religious practices are deeply disunified and heterogeneous, then similarities between their material remains are no guide to similarities in their cultural practices.

A third, and related, worry is about interpretation. The shift from rock art to cultural practice relies on inferring the art's meaning —and meaning is tricky. As Smith puts it:

Perhaps the greatest difficulty in getting at the meaning of prehistoric art is that we do not know the symbolic conceptions which were involved even in naturalistic representations. Are these to be taken literally, that is as signs? Or are they loaded symbols, part of a code to be broken? (1968, p.30). 
Human-made symbols admit many possible meanings - and getting an epistemic grip on these meanings is difficult. If there is no way for us to narrow the space of possible hypotheses, then we could be stuck at an empirical dead-end.

Note that the scope of these objections is unclear. Are archaeologists merely sounding a note of caution about the use of shamanistic ethnographies, are they claiming that such inferences are invalid in these particular circumstances, or are these applications of more general, in principle, arguments ethnographic analogy? Both direct and indirect analogies have come under three kinds of fire: the trustworthiness of the source, the idea that 'shamanism' is a good category, and the issue of interpretation. On the face of it, these objections tend towards the general rather than the specific —-there are overarching reasons to worry about ethnographic analogies which undermine particular uses. It is my task in the next two sections to shift such objections to the specific. As we shall see, if shamanistic analogies are problematic (or, for that matter, kosher), this needs to be shown via a detailed examination of the particular circumstance.

\section{Special Pleading?}

In this section I examine archaeological objections to ethnographic analogies in terms of the comparative method. There are two conclusions, first, there are no grounds for archaeological 'special pleading', that is, there is nothing prima facie different about the target of archaeological research — the archaeologist answers the same charges as the biologist. Second, license is provided on a case-by-base fashion. My aim here is neither to vindicate nor damn ethnographic analogy: I am neutral about the evidential worth of any particular instance of it. Rather, I am targeting what must be shown if ethnographic analogies are to be especially troublesome, and when they can be considered valid evidence. Special pleading could take two forms: first, there might be qualitative, in-principle differences between archaeological and biological targets; 
second, there could be qualitative differences: archaeological targets could be more intransigent epistemically speaking. I will discuss both.

My way into these questions is via a comparison with the comparative method, so it is worth making explicit their commonality: the comparative method and ethnographic analogies are instances of the same patterns of reasoning. The argument is summarized in table 1.

\begin{tabular}{|c|c|c|c|}
\hline & $\begin{array}{l}\text { Pattern of } \\
\text { Reasoning }\end{array}$ & $\begin{array}{c}\text { Targets } \\
\text { conceived as... }\end{array}$ & Validity turns on... \\
\hline $\begin{array}{l}\text { Homologous } \\
\text { Inference / } \\
\text { Direct } \\
\text { Ethnographic } \\
\text { Analogy }\end{array}$ & $\begin{array}{l}\text { Inference along } \\
\text { historical } \\
\text { continuity }\end{array}$ & Token-level & $\begin{array}{ll}\text { - } & \text { Relevant Similarity } \\
\text { - } & \text { Lability of trait } \\
\text { - } & \text { Temporal Distance }\end{array}$ \\
\hline $\begin{array}{l}\text { Homoplastic } \\
\text { Inference / } \\
\text { Indirect } \\
\text { Ethnographic } \\
\text { Analogy }\end{array}$ & $\begin{array}{l}\text { Inference via a } \\
\text { model linking } \\
\text { traits to other } \\
\text { features } \\
\text { (constrained to a } \\
\text { context) }\end{array}$ & Type-Level & $\begin{array}{ll}\text { - } & \text { Phylogenetic distance } \\
\text { - } & \text { Relevant similarity } \\
\text { - } & \text { Number of data-points }\end{array}$ \\
\hline
\end{tabular}

First, compare a homologous inference to a direct ethnographic analogy. According to the 'early hominid' hypothesis, $H$. floresiensis is habiline on the basis of their shared features, and the idea that it is plausible that a line of biological inheritance connects the two. According to the African shaman hypothesis, African rock art is explained by shamanistic practices, on the basis of the shared features of San ritual motifs and those from rock art, and the plausibility of a line of cultural inheritance between them. Both homologous inferences and direct analogies infer along lines of inheritance, their plausibility turns on (relevant) similarity and—and this is importanthow labile we ought to expect the feature in question to be.

Second, compare a homoplastic inference to an indirect ethnographic analogy. The 'late hominid' hypothesis treats $\mathrm{H}$. floresiensis as an erectine gone island dwarf, on the basis that, across the mammalian phylogenetic group, dwarfism correlates with the suite of traits expressed 
in $\mathrm{H}$. floresiensis. On the European shaman hypothesis, Palaeolithic rock art is explained in shamanistic terms, on the basis of a model that in humans (due in part to continuities in the perceptual effects of altered consciousness) shamanism correlates with features of rock art. Both homoplastic inferences and indirect ethnographic analogies work by supporting models linking features within certain constraints, that is, they are conceived on the type-level. Their plausibility is based on how likely the constraints are-that is, how similar ought we expect the critters in that group to be, how many independent data points there are for the model, and the level and type of similarity.

In terms of brute evidential reasoning, then, an ethnographic analogy is simply an example of the comparative method applied to archaeology. This is, I think, unsurprising: after all, Wylie (1985) has shown that ethnographic analogy can be understood in terms of the logic of analogous reasoning in general, and moreover I have operated at such a coarse grain of analysis that it is easy to unify things. However, I think important upshots emerge from this point. With a link established between archaeology and biology, we can re-examine and reconceptualise general objections to ethnographic analogy. By doing so, I show that such general objections are misplaced, and point to which details would help us ascertain the licence of a particular inference.

\subsection{Interpretation}

Archaeologists study the remains of incredibly complex critters who arrange themselves into very complex systems—societies—and one of the tricky things about these critters is their intentions: humans have goals, aims and reasons. This means that if material remains are to be inroads to past human lifeways, they need to be interpreted. But interpretation is difficult because human intention is so diverse. Yes, the Elan is a central motif in San shamanistic practices, representing the power of the entranced shaman, but why should I think this is true of 
a painting of an Elan—perhaps the painter just liked Elan, or Elan represented something else? Moreover, how we interpret the Elan is highly dependent on our background theories: if I am in a 'new archaeology' frame of mind I will approach the art asking what kinds of adaptive information could be contained within it, while if I am influenced by structuralism I might ask how it could serve to reinforce social order. What is interpreted depends vitally on facts about the interpreter.

Problems of interpretation are best understood as problems of underdetermination. Underdetermination is standardly a relationship between theories and evidence: two theories are underdetermined just in case there is insufficient evidence to discriminate between them. We can distinguish between 'in principle' underdetermination: where the empirical consequences of two theories are identical, and 'transient' underdetermination, where current evidence does not distinguish between hypotheses (Turner 2005, Stanford 2009, Sklar 1977). Surely archaeological hypotheses are not underdetermined in principle: presumably we do sometimes work out what the past intentions of human actors were. Problems of interpretation should be read as the worry that we don't have (and are not likely to get) enough evidence to satisfactorily empirically distinguish between the live options.

Problems of underdetermination are certainly not unique to archaeology. Consider the two competing paleoanthropological hypotheses from section 2. To claim that hobbits are erectine or habiline requires differing interpretations of the remains' features. That is, the features alone don't speak either way. It is only in virtue of background theory that material remains gain evidential relevance, no matter what the context. Biologists have developed criteria for spotting, say, when two traits are homologues or homoplasies. These criteria are rich and certainly not theoretically innocent; they must be interpreted, and some of the issues are extremely subtle. Does this halt progress in biology? It does not seem so. 
And so, if interpretation ought to be read in terms of underdetermination, there isn't any in principle special pleading on the behalf of archaeologists here. However, might there be something different in quantity, if not kind. That is, might underdetermination problems be particularly pressing for ethnographic analogies? Well, under what circumstances should we expect underdetermination to be particularly prevalent? Here are some thoughts.

First, notice that underdetermination has two sources. Call one source 'evidential' underdetermination. Here we know what would decide between our hypotheses, but the evidence just isn't available. Say we found evidence of a $\mathrm{H}$. habilis radiation, or a sequence of finds providing an evolutionary sequence from erectine ancestors to $\mathrm{H}$. floresiensis. That, probably, would decide the hobbit debate one way or the other. However, such conclusive evidence isn't available. Call the other 'midrange' underdetermination. I'm using 'midrange' in reference to Binford's 'middle-range theory' (Kosso 2000, Binford 1977); these are theories which grant observational reports evidential relevance. A paleobiological example of midrange theory is taphonomy, the science of fossilization. Fossils are evidentially relevant to extinct lineages in virtue of our understanding of fossilization formation which taphonomy provides. For LewisWilliams, it is in virtue of both the underlying psychological theory and the model connecting cave art to shamanism that South African San ethnography is evidentially relevant to Paleolithic society. If we are uncertain of our midrange theory, or such theory is incomplete, then we don't even know which observations could be decisive either way. If we didn't know how fossils formed, then it would be very difficult to know how to use aspects of fossil morphology to distinguish between different hypotheses about past lineages. This is because understanding fossil formation is often necessary to split the informative biological signal from features which are due to geological or environmental influence after the organism's death. I suspect that this kind of worry is the one which motivates archaeologists concerned about interpretation. That is, we do not have the requisite theories required to know what evidence could distinguish between various interpretive hypotheses. 
And so, the claim that ethnographic analogies are particularly prone to underdetermination could be driven by the thought that (1) good ethnographies would be great evidence, but they just aren't available (evidential underdetermination), or (2) we just don't have the theories required to link ethnographies to past human societies (midrange underdetermination). Interestingly, these options are intimately linked to the next two challenges I am to discuss. The first is about how good our evidence about ethnographies is, the second is about the kinds of systems human societies might be and how this affects the relevance of ethnographic data. If I am right, then complaints about interpretation actually either boil down to worries about uniformitarian principles_-human societies are just the wrong kinds of systems; or worries about sufficiency of evidence. Let's turn to those issues.

\subsection{Uniformitarianism}

There are a wide variety of uniformitarian principles. Basically, they tell us that a phenomena, force, or regularity from some domain also operates in another. In a sense, Newton's arguments for universal gravitation - that the mathematics representing forces on pulleys and weights also represented the relationships between celestial bodies-was uniformitarian. Typically, though, the term is related to $19^{\text {th }}$ Century geologists such as Lyell, who argued that we ought to use the kind of small-scale geological processes we see now (erosion, for instance) to explain geological form—a slow and steady approach to explanation which heavily influenced Darwin. Some kind of uniformitarian principle is necessary to license ethnographic analogy, but it is very important to get clear on what type.

Some complaints against ethnographic analogy assume that they require a kind of general uniformitarianism. Holly Hayter is a good example, she argues that: 
There are a number of problems involved in the use of ethnographic analogy. Basically, these problems lie in the various underlying uniformitarian, environmental-deterministic notions upon which the notion of analogy is based. (47-48).

In other words, analogies require uniformitarian-that is, deterministic — connections between environments and human culture. But there aren't deterministic connections between environments and human culture, again quoting Hayter:

... ethnographic studies have only proved that there are an incredible amount of different codes of behavior practiced by many groups throughout the world. There are insurmountable factors involved in structuring human behavior: no one practice can be narrowed down to environmental, social, or biological factors. There are no such things as cultural laws... (44-45).

Human cultural groups, then, are too complex for simple deterministic inferences from environment to culture to hold—human systems are path-dependent, interdependent, and highly context-sensitive. In virtue of this, such systems are not amenable to strict uniformitarian treatments. Hayter is right about this. Moreover, as we saw earlier, she and other archaeologists might also be right to put pressure on the notion that 'shaman' or 'hunter-gatherer' are good categories. They may be gerrymandered collections of disparate cultural and subsistence practices. However, there are two fundamental errors here: first, in thinking that ethnographic analogies (direct or indirect) require deterministic connections between environment and culture; second, that they require robust categories like 'hunter-gatherer'. To see why, let's reexamine the relationship between hobbits and hippos.

The homologous inference from $\mathrm{H}$. floresiensis's traits to a habiline ancestry relied on an uniformitarian principle: we could call this 'phylogenetic inertia' (Griffiths 1996, Levy \& Currie 2015). Phylogenetic inertia, taken generally, says that traits are likely to remain stable over time; it provides a general license to think biological traits are not (very) labile. Such a principle could be motivated by evolutionary theory. In order for complex traits to cumulatively evolve, avenues of 
inheritance must be fairly robust, and so we should expect inertia. Hopefully it is immediately obvious that this principle is more applicable for some traits than others, as inheritance channels differ in their robustness. This is why investigating the lability of a trait matters. Phylogenetic inertia, then, is no deterministic principle. The use of pygmy hippos to test the model of insular dwarfism in $\mathrm{H}$. floresiensis also didn't require that there be any deterministic relationships: things are more subtle than that. It produced evidence that the model of insular dwarfism applied to the hobbits was faulty.

And so, analogous evidence in no way requires commitment to strong deterministic principles-but, as we shall see in 5.2, working out how robust the relevant inheritance channels are (in the direct case) and how determinate the material-remain/cultural regularities are (in the indirect case) is extremely important for licencing an ethnographic analogy.

Moreover, using ethnographic analogies does not require robust unified categories like 'hunter-gatherer'. Remember, an ethnographic analogy links a material remain with a cultural product (or a cultural product with another) either through a model, or along lines of ancestry. In neither case do we need the categories to be unified in a deep way. Hippopotamus and Hominids are very different kinds of critters. We do not need for them to fall within some general category for their use in a homoplastic inference: they just need to be similar in the relevant respects. Even if there are enormous differences between so-called 'hunter-gatherer' groups, what matters for ethnographic analogy is the robustness of the relevant similarities.

The lesson here is that uniformitarian principles are applicable case-by-base. Some systems act in a relatively regular way. These systems (at least in regards to the way they are regular) are well behaved and uniformitarian. Others are more chaotic and such principles do not hold. Of course systems can be systematically chaotic: there is a whole range of ways in which regularities can hold across systems! And note that these features of systems are empirically investigable, at least in principle. 
So, if there is any special pleading on the part of archaeology about reliance on uniformitarian principles, this would require an argument that archaeological targets are more chaotic, irregular and labile than biological (or paleoanthropological) ones; there is no in-principle difference.

\subsection{Ethnographies are suspect}

Archaeologists have justifiable concerns about ethnographic data. Many are reports compiled by untrustworthy narrators-as Hiscock (2007) details, many of the sources for indigenous Australian ethnographies are from $19^{\text {th }}$ Century missionaries, hardly the most detached scientists (although some were remarkably even-handed and clear). Moreover, the reports are typically qualitative impressions, hardly hard data. Finally, they are carried out by anthropologists who have different needs and interests to archaeologists (an anthropologist conducting an ethnography is unlikely to pay attention to the connections between material culture and, say, religious practices). Holly Hayter, again, summarizes the problems. Ethnographic studies are (1) time-limited, (2) based on unreliable informants, (3) based on ambiguous and biased data collation:

Thus, any hypotheses born out of ethnographic data will not necessarily predict what has happened in prehistoric times but will more or less regurgitate what the ethnographers have stated (42).

This is a different complaint than that of the last section. There, we were concerned about whether our target system admitted of analogous treatments. Here, we are concerned about whether the evidence we have about that system is kosher. The first worry was, given some good ethnographic data, is that data relevant to our archaeological target. This second worry is whether the ethnographic data is good in the first place.

Historical scientists frequently work under conditions of apparent evidential paucity: it is not merely archaeological remains which are biased, ambiguous and fragmentary. Studies of $H$. 
floresiensis are marked by degraded, incomplete—and few-specimen. There are the remains of 13 incomplete hobbit skeletons, and this is a fairly rich find by the standards of paleoanthropology. In order for there to be special pleading, it must be that ethnographic data is highly biased, ambiguous and fragmentary. There could be something to this: although fossil remains of pygmy hippopotamus are incomplete, the hippos will not actively mislead you. In 5.1, I will defend the use of such data in reconstructing the past.

My aim thus far has been to clarify: just what challenges ethnographic analogies face, and what would it take for these challenges to be particular to archaeology. As we have seen, there are no epistemic issues unique to archaeological comparative data. Moreover, the license depends on facts on the ground. I want to shift to a more normative frame of mind in the last section. Here, I will discuss what archaeologists can (and sometimes in fact are) doing about these apparent problems.

\section{Local Licence and Comparative Data in Archaeology}

In the last section, I argued that concerns about ethnographic analogies boil down to two concerns: issues about evidence, its trustworthiness and stability, and issues about the regularity of human cultural behavior and systems. These must be understood to ascertain the evidential weight of an ethnographic analogy. Here, I will point to how historical scientists generally overcome these problems, and point to where they may, indeed, be problematic.

\subsection{Evidence \& Culture}

There are many ways in which historical evidence can be problematic. Downstream traces can degrade and so be incomplete. We can lack the required theory to link evidence to the past. Evidence can be biased. For ethnographic analogies, the worry is that the ethnographies themselves do not truly reflect human societies. And surely, some of the time, they do not, or do 
so incompletely. I want to make two, perhaps obvious, points about such situations. The first concerns methodology, and simply recommends collecting ethnographies that are not only better organized, but targeted. The second concerns the epistemic role ethnographic analogies play in archaeology.

Archaeologists have begun collecting their own, and testing, ethnographic data-and this is an obvious response to worries about ethnographic evidence. I want to point out a few things about this practice. Recall that indirect analogies (and surely most of these archaeological ethnographic studies will be indirect—-there are so few groups with direct connections remaining!) rely upon models that connect features of human life. Like in the biological case, these are ceteris paribus on two counts. First, they are not intended to hold across all cases. Martin et al's model is only intended to hold across mammals - there is no discussion of how dwarfism is developmentally expressed in birds or fish, for instance. Second, they allow exceptions—such models are only intended to hold across typical specimen (see Currie 2015).

This caveating is necessary for biologists because of the 'historicity' of biology ${ }^{11}$ - that is, biological systems are highly path dependent (natural selection, after all, can only work with what it has). This path dependence means that many regularities across biological systems will be highly constrained to particular ancestral groups_regularities, when they occur, will do so within shared histories. Potentially, human cultural groups could exhibit historicity writ large—they could be highly contingent, and admitting only of localized evidential treatment. But this needs to be shown. Showing that, for instance, there is no monolithic 'hunter-gatherer' culture does not show that there are no exploitable regularities across human groups.

And so, for direct analogies, we need to know how stable we should expect human culture to be. In some cases, not very: given the shear plasticity of human behavior and culture, I worry that direct analogies are often not useful. Homologous inferences gain their epistemic warrant, not

\footnotetext{
${ }^{11}$ For discussion of historicity in biology, see Beatty (2006) and Desjardins (2011)
} 
only from similarity, but because, for many biological traits, we expect robust inheritance channels. We expect this because natural selection requires such channels to build complex morphologies, and because developmental systems ensure it. Does something like this hold for human culture? Pessimism on this question may be too quick: human groups maintain channels of cultural inheritance by constructing 'epistemic niches' which ensure uptake of the right skills and beliefs across generations (Sterelny 2003). It may turn out that some aspects of cultural inheritance, particularly when buttressed by high fidelity channels (for instance, those maintained by song, story and ritual), are extremely robust. Regardless to say, I would like to see more study of which aspects of human life are stable over time, and which are not.

Here is one example of how we might empirically investigate the trustworthiness of ethnographies, and the robustness of cultural inheritance. Bernardini (2008) reports that Hopi informants are not only able to identify grave goods from a thousand year's old grave site, but also successfully predicated other objects found in the same assemblage. This doesn't show that continuity between the Hopi and the makers of the grave site is doing the work, of course (for instance, they could just be very familiar with such sites), but nonetheless presents an interesting example of how to test ethnographic reliability ${ }^{12}$.

For indirect analogies, we want to know how stable we should expect correspondences between environment and cultural traits to be. This, oddly enough, I have more optimism about: these correspondences needn't be determinate, they needn't be exceptionless. They just need to provide evidence. Given how adaptive and flexible human groups are, where there are good cultural solutions to problems, we should expect these to crop up often. Overall, then, collecting ethnographic data is important for answering such questions, and targeted investigations are called for.

\footnotetext{
${ }^{12}$ Thanks to Michelle Turner for the example.
} 
The lesson here is that whether we have good ethnographic evidence is an empirically tractable question. Archaeologists can study and generate ethnographic material in a way which leads to better understanding, and their applicability to reconstructions of past cultures.

The main problem, I think, with focusing on the evidential shortfalls of ethnographic analogies and then concluding that their use is non-evidential, is the nature of confirmation in historical science. Even if ethnographic analogy is weak evidence, historical science is all about drawing together different strands of weak evidence to build a surprisingly strong whole. Several philosophers have emphasized the importance of independent streams of evidence in the support of historical science, and it is worth summarizing that work here.

Alison Wylie $(2002,2011)$ distinguishes between two forms of independence. Vertical independence concerns the relationship between theories which play different roles. Horizontal independence concerns different types of evidence, converging on the same hypothesis. I will focus on horizontal independence.

Of necessity, evidential reasoning depends on multiple strands of arguments: it emanates from disparate elements of the archaeological record, draws on background knowledge that originates in diverse source fields, and bears on an array of conditions and events that constitute the complicated lives of the material things that make up the archaeological record (Wylie 2011 pp386-387).

Horizontal independence concerns evidence-streams playing the same role (i.e supporting the same hypothesis) from disparate sources. This is seen in the application of different dating techniques:

Consider, for example, evidential arguments that turn on the juxtaposition of measures of radiocarbon decay, magnetic orientation, tree ring counts, and stylistic variability over time (Ibid, 387). 
These different measures are independent, and data-convergence reassures us of their veracity. Forber \& Griffith (2011) make the same point, arguing that historical hypotheses are largely justified on these terms. They focus on dating using ${ }^{14} \mathrm{C}$ and tree-ring counting:

Whatever the insecurities inherent in each line of evidence, their congruence raises the credibility of the claims they support insofar as the conditions or assumptions that might produce error in a ${ }^{14} \mathrm{C}$ date are not the same as those that might bias a date based on tree ring sequences of rates of stylistic change (387-388).

According to Forber \& Griffith, the ability of historical scientists to draw on independent lines of evidence undercuts underdetermination problems. As evidence-lines converge, the total evidence for a past event increases. The rationale is that different factors are required to confound the different measurements. It would be highly unlikely for both ${ }^{14} \mathrm{C}$ data and tree ring data to screw up at the same time; and it is more unlikely still for their results to converge in spite of this. When horizontally independent lines of evidence converge, the hypothesis that both measurements are correct is much more likely than the hypothesis that both are false. This kind of reasoning applies to analogies as well: see my (2013) discussion of 'integrated explanations', which discusses explicitly how analogous and non-analogous information can aid in reconstruction.

The point of all this is to show that historical hypotheses can, in terms of evidential support, be more than the sum of their parts. If that is right, then it is a mistake to discount a line of evidence, particularly one as potentially important as ethnographies, because the evidence is somewhat problematic. Of course, it would be equally foolhardy to prioritize that evidence without good reason. Even if ethnographic information is sketchy, it can nonetheless provide an important line of evidence to support archaeological hypotheses.

\subsection{Cultural Reconstruction}


Some archaeological concerns about ethnographic analogy come from beliefs about the nature of human cultural systems. The objection that there is no unified, discrete 'shaman' category is one, and a related complaint is the apparent commitment to uniformitarian principles. As we saw in 4.2 , however, these objections need to be tempered: ethnographic analogy neither requires discrete categories nor strong uniformitarianism. However, different systems behave more or less regularly at different levels of description—and the effectiveness of an ethnographic analogy depends these features. This requires fine-grained multi-leveled examinations of ethnographic data. Moreover, in the last subsection we saw that historical reconstruction often involves combining different lines of evidence, and that the consilience of individually weak lines can make for robust hypotheses. Happily, Christine VanPool (2009) has started just this sort of analysis of Shamanistic practices, and pausing to consider her example is illustrative.

First, VanPool, drawing from anthropological surveys, agrees that 'Shaman' is not a discrete category: 'shamanistic' practices, individualized, idiosyncratic, and involving trance-states, grade into more organized 'priestly' practices in a non-systematic way. This is no block to the class being useful however, VanPool recommends taking shamanism to be a 'polythetic' class “... in which members share many, but not all of the defining characteristics" (179). This class shades into the polythetic class of priests:

As intuitive as it may seem, however, shamans and priests are not appropriate archetypes and do not reflect dichotomous or essentialist "types" in the sense that they are immutable states wholly distinct from one another. Instead they are analytically useful groupings that reflect the co-occurrence of religious traits that tend to correspond with one another as the level of cultural complexity shifts (178).

Second, VanPool analyses various features of Shamanism, and identifies which features of shamanistic systems are more likely to be universal, and those which are more particular. For instance, Shamans achieve altered states of consciousness through a variety of methods: 
psychoactive drugs, chant and ritual, sensory deprivation, and so forth. There are regularities about how these methods affect human perception, and these can make a difference to the material remains of shamanistic practices. To take one of VanPool's examples, nicotine intoxication effects color perception, excluding the palate to white, yellow and black, while peyote produces vivid 'psychedelic' experiences. These colors are reflected in shamanistic art associated with those drugs. However, although the perceptual experiences generated by different methods of achieving altered states are general, how these are understood is culturally variable.

Both entopic images and hallucinations are universal, but their utilization and interpretation by shamans... are culturally specific... Understanding the cultural filter used to interpret the hallucinations encountered during SSC should be central to the anthropology of religion, given that it reflects cultural transmission between the practitioners, aspects of a culture's cosmology, and their view of the spirit world (180).

Third, VanPool documents various material remains ('sacra') which are associated with shamanistic practices. These include imagery, including rock art, musical instruments, the remains of psychoactive plants, the tools associated with them (pipes for instance) and spaces put aside for shamanistic activities. VanPool emphasizes the importance of utilizing these material remains as independent lines of evidence for identifying shamanistic practices. For instance,

... most shamanistic rituals include some form of hallucinogenic agent. Shamanistic sacra will therefore be indicated by its association with the agents themselves (e.g., macrobotanical remains of datura) and the tools used to administer them (e.g., pipes for smoking tobacco). This can be compared with the imagery (e.g., colour symbolism, types of images depicted) to determine if they correspond with one another (183).

In short, it is a mistake to take archaeological evidence atomistically-rather, hypotheses need to be considered as a whole and the dependencies between different lines of evidence need to be considered. An assemblage consisting of botanical tobacco remains and pipes, as well 
as white, yellow and black imagery, points clearly towards tobacco-based shamanistic practices. This is because the assemblage would be less likely without the presence of shamanism.

VanPool uses ethnographic data to inform her reconstructions even though (1) the category 'shaman' is not discrete, (2) the anthropological data is sketchy, (3) human societies are complex and labile. She is able to do this by analyzing the ethnographic data in a fine-grained manner, allowing her to identify which features are likely to be common and which will be culturally specific, as well as by integrating the ethnographic data with analyses of material remains, the color-palate of rock artists, and psychological details of human perception. In VanPool's work, we see ethnographic analogy take its proper place as just one of our sources of information about the past. Understanding its limitations and integrating it with other sources is the secret to making ethnographic information relevant to archaeological reconstruction.

\section{Conclusion}

I have argued that ethnographic analogy just is the application of the comparative method to human culture and material remains. On this basis, I have revisited the objections archaeologists have raised to the use of analogy. I argued that all-encompasing rejection (or acceptance!) of ethnographic analogies should themselves be rejected. The action is local. Moreover, even when analogies are carried out on the basis of incomplete, biased ethnographies, and even when the target systems are highly sensitive to context and exhibit 'historicity', ethnographic data can play an important role in supporting archaeological hypotheses. They count as one line of evidence which archaeologists can exploit. Is there, then, cause for special pleading among archaeologists—are there systematic differences between archaeological method, or archaeological targets, and their biological analogues? No. In either case, the applicability of both inferences along inheritance channels, and models which exploit regularities, is piece-meal and 
particular. There is no such thing as the licence for ethnographic analogy, but nor is there such a thing as the objection to it.

\section{Bibliography}

Argue, D. Morwood, MJ. Sutikna, T. Jatmiko, Saptomo, EW. (2009). "Homo floresiensis: a cladistic analysis." Journal of Human Evolution 57(5): 623-639.

Bahn, P., 1997. Membrane and numb brain: a close look at a recent claim for shamanism in Paleolithic art. Rock Art Research 14, 62-68.

Beatty, J. (2006). Replaying life's tape. The Journal of philosophy, 336-362.

Bernardini, W. (2008). Identity as history: Hopi clans and the curation of oral tradition. Journal of Anthropological Research, 483-509.

Berrocal, M. 2011 Analogical evidence and shamanism in archaeological interpretation: South African and European Palaeolithic rock art. Norwegian Archaeological Review 44 (1): 1-20

Brigandt, I \& Griffiths, P (2007). "The importance of homology for biology and philosophy." Biol Philos 22: 633-641.

Bromham, L., \& Cardillo, M. (2007). Primates follow the 'island rule': implications for interpreting Homo floresiensis. Biology letters, 3(4), 398-400

Brown, P. Sutikna, T. Morwood, MJ. Soejono, RP. Jatmiko, Wayhy, Saptomo, E. Awe Due, Rokus. (2004). "A new small-bodied hominin from the Late Pleistocene of Flores, Indonesia." Nature 431(7012): 1055-1061.

Conkey, M.W \& Hastorf, C. A (ed) 1990. The uses of style in archaeology. Cambridge: Cambridge University Press 
Chippindale, C., \& Taçon, P. S. (Eds.). (1998). The archaeology of rock-art. Cambridge University Press.

Currie, A (2014a). Venomous Dinosaurs and Rear-Fanged Snakes: Homology and Homoplasy Characterized. Erkenntnis 79 (3):701-727.

Currie, A (2014b). Marsupial lions and methodological omnivory: function, success and reconstruction in paleobiology. Biology \& Philosophy, 1-23. (10.1007/s10539-014-9470-y)

Currie, Adrian (2013) Convergence as Evidence. Br J Philos Sci 64 (4): 763-786.

Desjardins, E. (2011). Historicity and experimental evolution. Biology \& philosophy, 26(3), 339364.

González-Urquijo, J., Beyries, S., \& Ibáñez, J. J. (2015). Ethnoarchaeology and Functional Analysis. In Use-Wear and Residue Analysis in Archaeology (pp. 27-40). Springer International Publishing.

Griffiths, P. E. (1996). The Historical Turn in the Study of Adaptation, The British Journal for the Philosophy of Science, 47, pp. 511-32.

Hall, B. K (2007). Homoplasy and homology: dichotomy or continuum? J Hum Evol. 2007 May;52(5):473-9.

Hall, B. K. (2003). Descent with modification: the unity underlying homology and homoplasy as seen through an analysis of development and evolution. Biological Reviews of the Cambridge Philosophical Society, 78(3), 409-433.

Hayter, H (1994). Hunter-gatherers and the Ethnographic Analogy: Theoretical Perspectives. Totem: The University of Western Ontario Journal of Anthropology. 1(1).

Hiscock, P. (2007). Archaeology of ancient Australia. Routledge.

Jacob, T., Indriati, E., Soejono, R. P., Hsü, K., Frayer, D. W., Eckhardt, R. B., ... \& Henneberg, M. (2006). Pygmoid Australomelanesian Homo sapiens skeletal remains from Liang Bua, Flores: 
Population affinities and pathological abnormalities. Proceedings of the National Academy of Sciences, 103(36), 13421-13426.

Jungers, WL. Harcourt-Smith, WEH. Wunderlich, RE. Tocheri, MW. Larson, SG. Sutikna, Due, RA. Morwood, MJ. (2009). "The foot of Homo floresiensis." Nature 459(7243): 81-84.

Layton, R. (2000). Shamanism, totemism and rock art: Les chamanes de la préhistoire in the context of rock art research. Cambridge archaeological journal, 10(01), 169-186.

Lieberman, D. E. (2009). Palaeoanthropology: Homo floresiensis from head to toe. Nature, 459(7243), 41-42.

Lewis-Williams, J. D. (1995). Seeing and construing: the making and 'meaning' of a southern African rock art motif. Cambridge Archaeological Journal, 5(01), 3-23

Lewis-Williams, J. D. (1991). Wrestling with Analogy: A Methodological Dilemma in Upper Palaeolithic Art Research. . Proceedings of the Prehistoric Society, 57, pp 149-162.

Levy, A \& Currie, A (2014). Model Organisms and Not (Theoretical) Models. British Journal for the Philosophy of Science. Online first, doi:10.1093/bjps/axt055

McCall, G. S. (2007). Add shamans and stir? A critical review of the shamanism model of forager rock art production. Journal of Anthropological Archaeology, 26(2), 224-233.

Martin, RD. Maclarnon, AM. Phillips, JL. Dussubieux, L. Williams, PR. Dobyns, WB. (2006). "Comment on "The Brain of LB1, Homo floresiensis"." Science 312(5776): 999b-.

Montgomery, S. H., \& Mundy, N. I. (2013). Parallel episodes of phyletic dwarfism in callitrichid and cheirogaleid primates. Journal of evolutionary biology, 26(4), 810-819. 
Morwood, M. J., Brown, P., Jatmiko, T. S., Saptomo, E. W., Westaway, K. E., Due, R. A., ... \& Djubiantono, T. (2005). Further evidence for small-bodied hominins from the Late Pleistocene of Flores, Indonesia. Nature, 437(7061), 1012-1017.

Pearce, T. (2012). Convergence and parallelism in evolution: A Neo-Gouldian account. The British Journal for the Philosophy of Science, 63, 429-448.

Powell, R. (2012). Convergent evolution and the limits of natural selection. European Journal for Philosophy of Science, 2(3), 355-373.

Ramsey, G., \& Peterson, A. (2012). Sameness in biology. Philosophy of Science, 79(2), 255-275.

Ravn, M. (2011). Ethnographic analogy from the Pacific: just as analogical as any other analogy. World Archaeology, 43(4), 716-725.

Bednarik, R. G. (2012). Ethnographic analogy in rock art interpretation. Man In India, 91(2), 223-234.

Remane, A. 1952. Die Grundlagen des natu"rlichen Systems, der vergleichenden Anatomie und der Phylogenetik. Akademische Verlagsgesellschaft, Leipzig.

Smith, P. E. (1968). Problems and possibilities of the prehistoric rock art of northern Africa. African Historical Studies, 1-39.

Solomon, A. 1998. Ethnography and method in southern African rock art research. In Chippindale, C. \& Taçon, P. (eds). The Archaeology of Rock Art, pp. 268-284. Cambridge University Press, Cambridge.

Sklar, L (1977). What might be right about the causal theory of time. Synthese 35 (2):155 - 171 Stanford, Kyle (2009). Underdetermination of Scientific Theory. In Edward N. Zalta (ed.), The Stanford Encyclopedia of Philosophy 
Sterelny, K. (2003). Thought in a hostile world: The evolution of human cognition.

Thompson, R. H. (1956). The subjective element in archaeological inference. Southwestern Journal of Anthropology, 327-332.

Turner, D (2005a). Local Underdetermination in Historical Science. Philosophy of Science 72 (1):209-230.

VanPool, C. S. (2009). The signs of the sacred: identifying shamans using archaeological evidence. Journal of Anthropological Archaeology, 28(2), 177-190.

van Gijn, A L., \& D. Raemaekers (1999). Tool use and society in the Dutch Neolithic: the inevitability of ethnographic analogies. IN: Ethno-analogy and the reconstruction of prehistoric artefact use and production, Owen \& Poor (eds). Mo Vince Verlag, pp 43-52

Weston, E. M., \& Lister, A. M. (2009). Insular dwarfism in hippos and a model for brain size reduction in Homo floresiensis. Nature, 459(7243), 85-88.

Wimsatt, W. C. (1986). Developmental constraints, generative entrenchment, and the innateacquired distinction. In Integrating scientific disciplines (pp. 185-208). Springer Netherlands.

Whitaker, A. R., \& Tushingham, S. (2014). A Quantitative Assessment of Ethnographically Identified Activity Areas at the Point Saint George Site (CA-DNO-11) and the Validity of Ethnographic Analogy. Journal of California and Great Basin Anthropology, 34(1), 1-15.

Wong, K. (2009). Rethinking the hobbits of Indonesia. Scientific American,301(5), 66-73.

Wylie, Alison (2011). Critical distance : stabilising evidential claims in archaeology. In Philip Dawid, William Twining \& Mimi Vasilaki (eds.), Evidence, Inference and Enquiry. Oup/British Academy. 
Wylie, Alison (2010). Archaeological facts in transit: the "Eminent Mounds" of Central North America'. In How Well do 'Facts' Travel?: the dissemination of reliable knowledge, eg. P. Howlett and M.S Morgan (Cambridge, Cambridge University Press), pp, 301-22

Wylie, Alison (2000). Rethinking unity as a working hypothesis for philosophy of science: how archaeologists exploit the disunity of science. Perpectives on science, 7(3): 293-317

Wylie, A. (1985). The reaction against analogy. Advances in archaeological method and theory, 63-111. 